\title{
Nocardia cerebral abscess: new concepts in diagnosis, management, and prognosis
}

\author{
E. B Y R N E, B. P. B R O P H Y, A N D L. V. PERR E T T \\ From the Neurology and Neurosurgery Units, Royal Adelaide Hospital, Adelaide, South Australia
}

\begin{abstract}
S U M M A R Y Three cases of multiple cerebral nocardial abscess are presented. All were cured by a combination of chemotherapy and surgery, a unique experience. Early detection, appropriate chemotherapy, absence of underlying immune malfunction, and surgically remediable disease are good prognostic indices in cerebral nocardiosis. If other adverse prognostic factors are absent, however, multiple abscess formation does not preclude the possibility of cure. Accurate localisation of nocardia cerebral abscesses by computerised axial tomography is a great help in management if multiple lesions are present.
\end{abstract}

Cerebral nocardiosis is an uncommon and usually fatal infection, some 50 cases having been documented of whom only one-fifth have survived. Krueger et al. reviewed the literature in 1954 and noted that of 18 cases of cerebral nocardiosis reported to that date, only one, a case of meningitis, had survived the illness. Occasional cures have been reported since then but have not been sufficiently frequent to dim the pessimistic prognosis usually given to cerebral nocardiosis. Nocardial organisms are opportunistic pathogens which usually produce infection in immunocompromised hosts, and $75 \%$ of cases in the United States occur in this group (Beaman et al., 1976). Cerebral nocardiosis occurs less commonly in patients with an intact immune system who do not have an underlying disorder, such as disseminated carcinomatosis, which is likely to prove fatal in itself. Prognostic information in this group is limited and the main purpose of this report is to clarify this area. Our recent experience with cerebral nocardiosis is presented in which three cases all with multiple abscess cavities survived the illness. These cases, together with the other published reports of cured nocardia cerebral abscess, are analysed and favourable prognostic features reviewed.

\section{Case reports}

CASE 1

A 44 year old Caucasian man was admitted to the

Address for reprint requests: Dr E. Byrne, Neurology Unit, Royal Adelaide Hospital, North Terrace, Adelaide, South Australia 5000.

Accepted 17 April 1979
Royal Adelaide Hospital under the care of $\mathrm{Mr}$ T. A. R. Dinning for investigation of focal seizures involving his right face, impairment of speech, and right faciobrachial weakness all of recent onset. He had a past history of chronic bronchitis and had a heavy alcohol intake. He was afebrile and abnormal findings were confined to the central nervous system. A mild right hemiparesis and facial weakness were noted. There was no papilloedema or evidence of meningism.

Blood haemoglobin level was $13.5 \mathrm{~g} / \mathrm{dl}$, white blood count 10000 cells $/ \mathrm{mm}^{3}$, erythrocyte sedimentation rate $93 \mathrm{~mm} /$ hour. Liver function tests were normal. Chest radiography showed consolidation in the anterior segment of the right lower lobe but no organism was cultured from the sputum. A nuclear brain scan $\left({ }^{99} \mathrm{Tc}\right)$ showed an increased uptake of radionuclide in the left parietal area. Left carotid angiography and pneumoencephalography were considered to be within normal limits. A provisional diagnosis of left parietal neoplasm was made and craniotomy undertaken (surgeon Mr J. B. North). At operation an abscess cavity was found and aspirated, and a subtemporal decompression carried out. Nocardia species were identified in the aspirate so sulphadiazine $1 \mathrm{~g}$ six hourly was started immediately. Dysphasia and hemiparesis remitted completely within six weeks. Chemotherapy was stopped after six months. He presented again one year after the cessation of sulphadiazine with paresis of the left leg. Isotope brain scan $\left({ }^{99} \mathrm{Tc}\right)$ showed a small right parasagittal focus of nuclide uptake and some enlargement of the original left parietal 
focus. This was attributed to extension of the Nocardia asteroides infection and sulphonamide therapy was restarted and maintained for four years. Serial brain scans showed a steady decrease in the size of both lesions, and four years later the isotope brain scan was completely normal. Paresis of the left leg rapidly improved. The patient continued to drink alcohol heavily and died six years after presentation. At necropsy the meninges were adherent to the left cerebral cortex and reactive gliosis was noted at the site of the original abscess cavity. No evidence of persistent nocardia infection was found on histological examination. Gross cardiomegaly was noted, and death was attributed to congestive heart failure probably related to abuse of ethanol.

CASE 2

A 43 year old Caucasian woman was admitted to the Royal Adelaide Hospital under the care of the Professorial Medical Clinic and subsequently transferred to the care of Mr T. A. R. Dinning. She had a three week history of continuous headache, nausea, and vomiting, and over the week preceding referral had developed a progressive right hemiparesis. She had a heavy alcohol intake and a past history of treated hypothyroidism. A mild pleuritic illness four months earlier had been followed by malaise, weight loss, and night sweats. Chest radiography at that time was said to show consolidation of a lung.

On admission she was febrile $\left(38.8^{\circ} \mathrm{C}\right)$, disorientated in time and place, and had mild meningism. Severe expressive dysphasia and right hemiparesis were noted. Both plantar responses were extensor. The chest was clinically clear.

Initial investigations showed a haemoglobin level of $14 \mathrm{~g} / \mathrm{dl}$ and white blood count of 10500 cells $/ \mathrm{mm}^{3}$. A chest radiograph showed consolidation in the posterior basal segment of the left lower lobe. Plain skull radiographs were normal. Computerised axial tomography of the head revealed multiple low density lesions with enhancing peripheries consistent with multiple abscess cavities although metastases were also considered (Fig. 1). A lumbar puncture was performed using a small needle. Turbid CSF was encountered with 580 lymphocytes, 310 polymorphonuclear cells, and 10 red blood cells $/ \mathrm{mm}^{3}$. The CSF protein was $1.17 \mathrm{~g} / 1$ and glucose $3 \mathrm{mmol} / 1$. Cultures of CSF were negative after two days, and empirical treatment with penicillin and chloramphenicol was begun.

Further investigation was started. Bronchoscopy (Dr R. Antic) revealed mucosal oedema at the orifice of the left posterior basal bronchus.

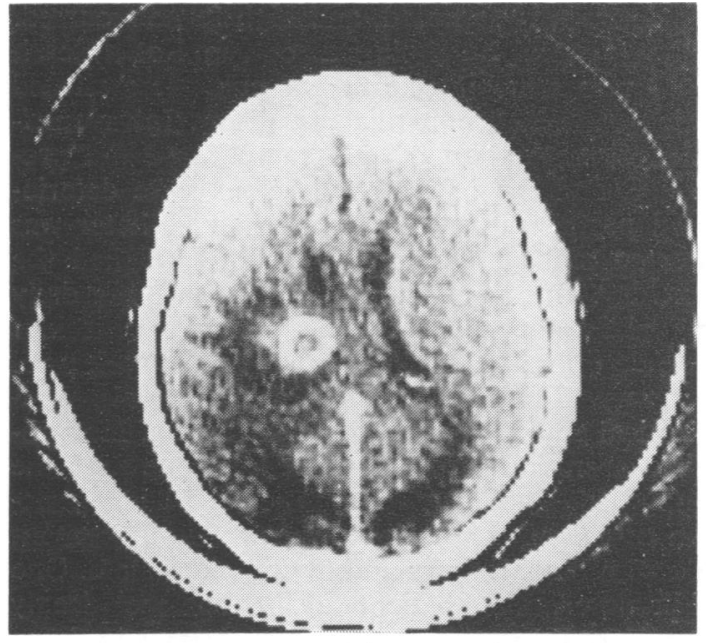

(a)

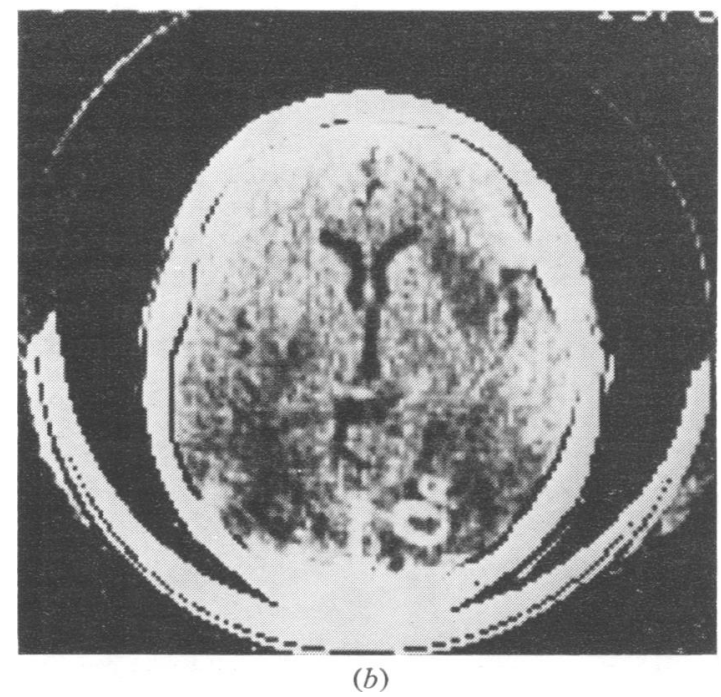

Fig. 1 Multiple abscesses in left parietal (a) and right occipitoparietal region (b). The ring shadows are caused by enhancement of the capsules and are surrounded by oedema, particularly the left sided lesions. There is further oedema in the right frontal region above another abscess (cut not shown).

No endobronchial lesion was seen. Mucosal biopsy samples were taken and the posterior basal bronchus brushed and washed. Examination of the histology revealed small numbers of branching Gram positive filamentous hyphae which were acid fast. Nocardiosis was diagnosed with reasonable certainty, and co-trimoxazole, six tablets per day, begun. After a week of treatment no definite 
improvement was noted, and high fever recurred. A left parietal burrhole was cut, and at a depth of $70 \mathrm{~mm}$ a small quantity of pus was aspirated which contained nocardia organisms. Intraventricular dissemination of pus complicated the procedure, and the patient's conscious state deteriorated postoperatively. A ventricular catheter was inserted and an Ommaya reservoir attached the next day. Intraventricular gentamycin $8 \mathrm{mg}$ daily was given for a week. The patient's conscious state deteriorated further over the next week until she was unresponsive to oral commands. Biochemical studies suggested a syndrome of inappropriate secretion of antidiuretic hormone with the serum sodium falling to $120 \mathrm{meq} / \mathrm{l}$. Fluid restriction to $500 \mathrm{ml} /$ day resulted in rapid improvement in serum biochemistry and in conscious state. One week later, the patient again became deeply comatose. Repeat CAT scan demonstrated a large haematoma in the right frontal region along the catheter tract (Fig. 2). This was decompressed surgically (Mr P. G. Carney) and later the encapsulated haematoma was totally excised. Co-trimoxazole therapy was continued and over the next month the patient gradually became more alert. Serial CAT scans (Fig. 3) showed resolution of remaining cerebral abscesses but communicating hydrocephalus developed and the insertion of a permanent shunt was necessary. Further immunological studies, including $T$ cell function tests, revealed no abnormality of cellular or humoral immunity.

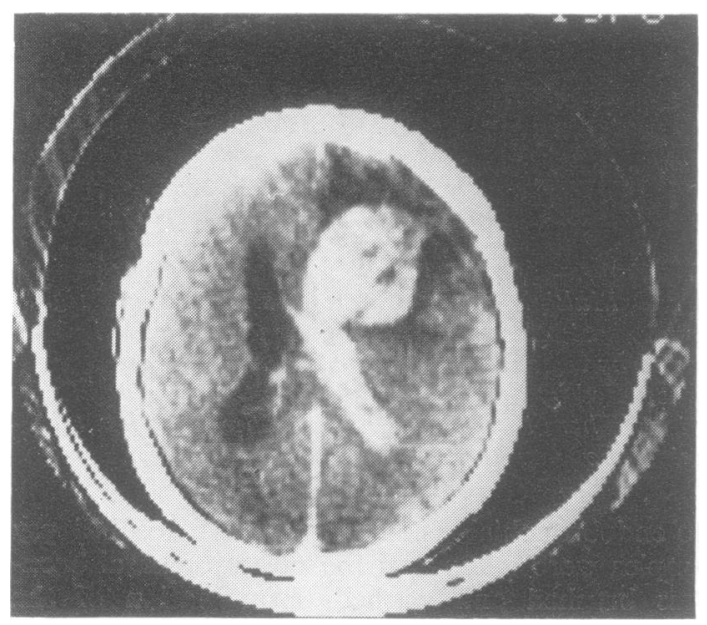

Fig. 2 One month later. The ventricular system has enlarged and there is a large right frontal haematoma which has extended into the right lateral ventricle. There is extensive frontal oedema around the haematoma and shift of midline structure to the left.

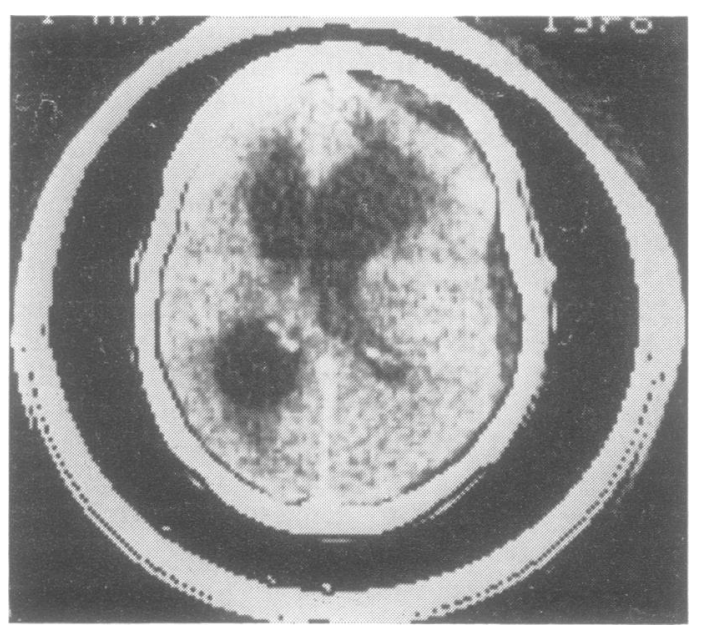

Fig. 3 Three months after admission. Further ventricular enlargement has occurred with displacement to the left by right frontal and temporoparietal surface fluid collections. There is only a small area of residual enhancement above the body of the left lateral ventricle.

The patient is well eight months after her presentation and is living with her family in a rural area. Co-trimoxazole therapy will be continued for two years.

\section{CASE 3}

A seven year old Caucasian boy was admitted to the Adelaide Children's Hospital and subsequently transferred to the care of Mr D. A. Simpson. For one week previously he had noted clumsiness in the left hand. A fluctuant abscess in the right buttock was drained, and the pus reported to be sterile. One year earlier he had had a febrile illness diagnosed as viral meningoencephalitis. For six months before presentation he had complained of headache, backache, and intermittent visual disturbance.

Examination on admission revealed gross bilateral papilloedema with concentric constriction of the left visual field and diminished vision in the right eye with perception of hand movements only. Left hemiparesis and left sided sensory ataxia were decumented. The left plantar response was extensor. A chest radiograph was normal. An echogram demonstrated shift of the brain to the left, and on electroencephalography a slow wave focus in the right parietal region was noted. A right parietal burrhole (Mr D. A. Simpson) was cut, and an encapsulated abscess was found. Two millilitres of yellow pus were aspirated and found to contain Nocardia asteroides. A left frontal burr- 
hole was cut under the same anaesthetic in an endeavour to reduce the intracranial pressure and enable a right osteoplastic craniotomy to be performed more easily. A second abscess in the left frontal area was found while attempting to tap the left frontal horn. After an infusion of urea, a large right lateral decompression was carried out. Sulphadiazine was continued postoperatively but over the next eight weeks the right lateral decompression increased in size and left hemiparesis worsened. Pneumoencephalography demonstrated an indentation in the left frontal horn and evidence of a much larger mass in the right frontoparietal area. An attempt was then made to enucleate the right sided abscess. At operation four abscesses were found in the right hemisphere, extending from the parietal area to the frontal pole. All were removed, a number of major middle cerebral vessels being sacrificed during the procedure. Postoperatively the patient made an excellent recovery and the left frontal abscess resolved with long-term sulphadiazine therapy. Considerable sensory and motor deficit, however, has persisted in the left arm and the left hand is of limited functional utility. A titanium cranioplasty was performed two years later, and review four years after presentation showed the patient to be in good health.

\section{Discussion}

As survival in patients with cerebral nocardiosis is unusual, it is surprising that no fatalities were encountered in three cases recently treated. In the first case, aspiration of the abscess coupled with sulphonamide therapy produced an excellent initial response. Relapse occurred one year after discontinuation of sulphonamide drugs, confirming the sometimes indolent nature of cerebral nocardial infection and the need for a prolonged course of antimicrobial treatment. Fortunately reinstitution of sulphonamides led to rapid resolution of symptomatology and normalisation of the technetium brain scan. It is possible that a neurosurgical approach could have been avoided in this case if efforts to isolate an organism from the lung had been successful.

In the second case, an attempt to needle one of the abscess cavities led to intraventricular dissemination of organisms, nocardial meningitis, and later communicating hydrocephalus. Accumulation of a haematoma around the ventricular catheter tract, necessitating drainage, was an additional complication. Meanwhile continued antimicrobial therapy led to resolution of residual abscess cavities. In retrospect, it is possible that in this case surgery may have been premature and some added disability resulted from it. The invaluable information provided by computerised axial tomography (CAT) scanning of the head is exemplified by this case. Widely disseminated multiple lesions were apparent on the first scan, discouraging any attempt at radical surgical removal. Cerebral haemorrhage and later hydrocephalus were identified early by CAT scanning, enabling definite treatment to be undertaken without delay. It is possible that this patient would not have survived without the accurate serial visualisation of pathology provided by tomodensimetry.

Advanced intracranial hypertension with visual loss in our third case necessitated early surgical decompression and partial excision of the abscess cavities. A residual left frontal abscess resolved completely with antimicrobial drugs. Symptoms of intracranial infection were present in this patient for at least six months before presentation and it is possible that earlier diagnosis and institution of sulphonamides might, in view of the excellent response eventually made, have reduced the residual disability. It is essential that the diagnosis of nocardiosis be established as early as possible, enabling time for a reasonable trial of chemotherapy to be given before intracranial hypertension necessitates radical neurosurgical intervention.

It is constructive to review the clinical features of the three cases presented here and of 13 other surviving cases of cerebral nocardiosis reported in the literature (Table) and to contrast these with some fatal cases. With two exceptions, all surviving cases received antimicrobial therapy specific for nocardia organisms. One case with unusual antimicrobial susceptibility received only penicillin and aureomycin (Krueger et al., 1954), and one penicillin and streptomycin (Munslow, 1954). There is no doubt that the latter cases suffered from nocardiosis and not from actinomymosis, and presumably surgery rather than penicillin therapy was curative in each. Where it is recorded, the duration of sulphonamide therapy has varied from eight weeks to several years. The fact that cases given shorter courses do not relapse uniformly suggests that the indolence of cerebral nocardiosis is variable. None of the cured cases had serious underlying disease such as lymphoma or carcinomatosis, a striking contrast with the fatal group. The extent of infection was relatively limited in most of the cured cases. Seven did not have clinically apparent pulmonary disease, which is unusual as the lung is the usual portal of entry for Nocardia asteroides (Frazier et al., 1975). 


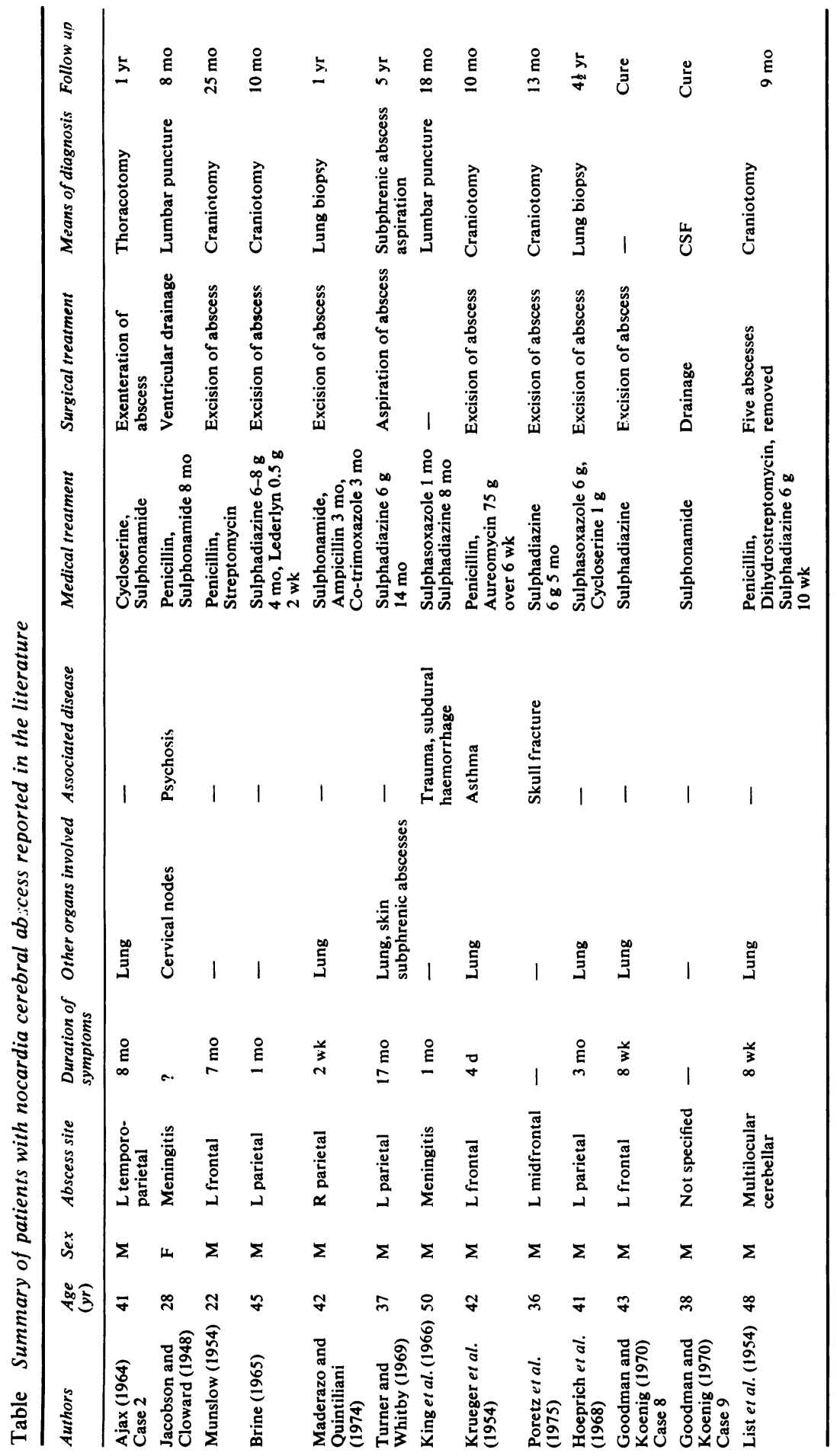


Multiple cerebral abscesses, with the exception of the three cases documented here, are uncommon in cured cases and if present usually represent a major abscess with several satellite lesions. That our cases did survive, however, suggests that although the prognosis in cases with multiple dispersed cerebral abscesses is bad, it is not uniformly hopeless if other bad prognostic indices are absent. Finally, all the cured cases listed underwent a neurosurgical procedure. In the majority a single abscess amenable to radical extracapsular excision was present, and this operation was performed. However, simple aspiration led to cure in one case (Turner and Whitby, 1969), and our experience confirms that total surgical removal of all infected material is not always necessary to achieve cure as this was not attempted in any of the three cases presented. In cases 1 and 2 sizeable abscess cavities were followed with ${ }^{99} \mathrm{Tc}$ scans in one case and with CAT scans in the second to complete resolution on antimicrobial therapy.

Consideration of some fatal cases of cerebral nocardiosis enables ready contrast with the successfully treated group. Often the diagnosis has been made preterminally or at necropsy (Krueger et al., 1954) or specific chemotherapy has been started only a few days before death (Pizzolato et al., 1961; Lee et al., 1963). Some of the fatal cases did not receive any specific nocardial chemotherapy even when a neurosurgical approach was made on an abscess (Teleghani-Far et al., 1964). Stevens' case (1953) underwent several neurosurgical procedures but was not given sulphonamides. In other cases, an inadequate course of sulphonamides has been given after early diagnosis, resulting in clinical relapse and death as in two cases of Murray et al. (1961). As might be expected, therefore, the prognosis is poor in patients who are diagnosed late in their course, in cases who do not receive specific chemotherapy and in cases in whom appropriate antimicrobial treatment is discontinued prematurely. Early diagnosis and appropriate treatment do not, however, ensure success (Turner, 1954). In reports of fatal cases the frequent occurrence of an underlying illness with grave prognostic implications is striking. Disseminated carcinomatosis is a common finding. The case of Arroyo et al. (1977) had disseminated renal carcinoma, case 5 in Larsen's series had breast carcinomatosis (Larsen et al., 1959), and case 3 of Adams et al. (1971) had Hodgkin's disease. It has been estimated that three-quarters of all cases of nocardiosis in America occur in immunodepressed hosts (Beaman et al., 1976), and it has been reported in patients receiving steroid and cytotoxic therapy after renal transplantation (Back et al., 1973a), and cardiac transplantation (Krick et al., 1975), in chronic granulomatous disease (Breed et al., 1948), dysgammaglobulinaemia (Neu et al., 1967), as well as complicating malignant disease (Young et al., 1971). It has been suggested that immunodepression is a bad prognostic factor in nocardia infections of any sort (Presant et al., 1973), and certainly this is the case in cerebral infection.

In many fatal cases nocardial infection has been widespread, involving multiple organs (Carlile et al., 1963; Adams et al., 1971; Arroyo et al., 1977). Multiple cerebral abscesses are commonly found in cases coming to necropsy (Stevens, 1953; Cupp et al., 1960; Murray et al., 1961; Shuster et al., 1967) or any abscess may involve vital brainstem structures as in case 3 in the series of Adams et al. (1971). In one earlier report, complicating subdural haemorrhage proved fatal which today might have been diagnosed more rapidly with CAT scanning and treated effectively (Pizzolato et al., 1961).

Good prognostic features in cerebral infection are, therefore, early diagnosis, administration of appropriate chemotherapy, restricted disease and, most important of all, the absence of underlying chronic illness. Although the outlook is better in surgically treatable cases, our personal series suggests that if the other favourable prognostic features are present, widely disseminated intracranial disease does not preclude the possibility of cure.

Diagnosis of nocardiosis may be difficult, as the large number of cases identified at necropsy testifies, and can only be achieved by identification of organism in tissue sections or in culture media. Bacteriological pitfalls have been discussed elsewhere (Fetter et al., 1967). In the usual situation where pulmonary and cerebral infection coexist, every effort should be made to obtain diagnostic material from the lung. Bronchoscopy, percutaneous lung biopsy, and transtracheal aspiration may all be necessary (Frazier et al., 1975). Needling of a cerebral mass lesion in confirmed pulmonary nocardiosis is not usually indicated for diagnosis and may be followed by meningeal contamination as in our second case and elsewhere (Turner, 1954; Kremer, 1972).

Accurate localisation of nocardia cerebral abscesses is a great aid in management if multiple lesions are present. In case 2 early CAT scanning showed multiple abscesses and excluded radical extracapsular excision as a practical therapeutic alternative. In case 3 , by contrast, who was treated before the CAT scan era, a left sided 
abscess cavity was encountered unexpectedly in the process of an attempted ventricular tap. Computerised tomography also has the advantage that it can be repeated easily, allowing close and accurate radiological monitoring of the response to therapy. In nocardia abscess, plane CAT scan cuts usually show an area of decreased density at the centre of the lesion. Sometimes the abscess cavity is visualised as a ring of increased density between the low density centre and low density oedema in the adjacent white matter. It is essential that intravenous contrast medium be given after the plane cuts as more than $80 \%$ of abscesses show a ring of enhancement (Schiefer and Huk, 1976). If no enhancement occurs, presumably a capsule has not yet been formed and the appearances are caused by a focal suppurative cerebritis. The case of nocardia abscess reported by Lott et al. (1977) did not show a ring shadow but contrast medium was not given. Three cases described by Claveria et al. (1976) all showed enhancement, two with ring shadows and the other with multiple uniformly enhancing lesions. It is sometimes difficult to distinguish between abscess and glioma or metastases with scanning. An abscess generally has a smooth capsule of uniform thickness as distinct from the more irregular margin of a neoplasm. Also, the contents of an abscess are usually of lower density than the central area of a necrotic glioma. Serial CAT scanning is the best way to follow patients with intracranial abscess, however treated (Claveria et al., 1976), and enables the attending physician to monitor therapy more closely than was possible in the past.

The best therapy in cerebral nocardiosis involves a judicious use of antibiotics and of neurosurgical intervention. Sulphonamides were the antimicrobial drugs of choice for many years and some authors still dispute that more recently developed drugs offer any additional advantages (Goodman and Koenig, 1970; Krick et al., 1975). Minocycline which is useful in pulmonary infections (Back et al., 1973b) does not cross the blood-brain barrier easily and should not be used alone in cerebral disease. Co-trimoxazole is now the agent of choice in cerebral nocardiosis. Both trimethaprim and sulphamethoxazole diffuse readily across the blood-brain barrier, achieving high CSF levels (Maderazo and Quintiliani, 1974). To ensure against the ever present risk of relapse, Goodman and Koenig (1970) suggest that chemotherapy be continued for at least 12 months and the late relapse noted in case 1 supports this contention.
We wish to thank Mr T. A. R. Dinning, Mr D. A. Simpson, and Mr P. G. Carney for permission to report these patients, and Dr J. F. Hallpike for helpful advice in compiling this report.

\section{References}

Adams, A. R., Jackson, J. M., Scopa, J., Lane, G. R., and Wilson, R. (1971). Nocardiosis: diagnosis and management with a report of three cases. Medical Journal of A ustralia, 1, 669-675.

Ajax, E. T. (1964). Acquired dyslexia: a comparative study of two cases. Archives of Neurology (Chicago), 11, 66-72.

Arroyo, J. C., Nichols, S., and Carroll, G. F. (1977). Disseminated Nocardia cavae infections. American Journal of Medicine, 62, 409-412.

Black, M. C., Adler, J. L., and Breman, J. (1973a). Influence of rejection therapy on fungal and nocardial infections in renal transplant patients. Lancet, 1, 180-184.

Black, M. C., Monaco, A. P., and Finland, M. (1973b). Pulmonary nocardiosis. Therapy with minocycline and with erythromycin plus ampicillin. Journal of the American Medical Association, 224, 1378-1381.

Beaman, B., Burnside, J., Edwards, B., and Causey, W. (1976). Nocardial infection in the United States 1972-74. Journal of Infectious Diseases, 134, 286289.

Breed, R. S., Murray, E. G. D., and Hitchens, A. P. (1948). Bergey's Manual of Discriminative Bacteriology, sixth edition, p. 897 . Williams and Wilkins: Baltimore.

Brine, J. A. S. (1965). Human nocardiosis, a developing clinical picture. Medical Journal of Australia, 1, 339.

Carlile, W. K., Holley, K. E., and Logan, G. B. (1963). Fatal acute disseminated nocardiosis in a child. Journal of the American Medical Association, 184, 477-480.

Claveria, L. E., Du Boulay, G. H., and Moseley, I. F. (1976. Intracranial infections: investigations by computerised axial tomography. Neuroradiology, 12, 59-71.

Cupp, C. M., Edwards, W. M., Walton, M. E., and Cleve, E. A. (1960). Nocardiosis of the central nervous system. Report of two fatal cases. Annals of Internal Medicine, 52, 223-226.

Fetter, B. F., Klintworth, G., and Hendry, W. S. (1967). Mycoses of the Central Nervous System, p. 145. Williams and Wilkins: Baltimore.

Frazier, A. R., Rosenow, E. C., and Roberts, G. D. (1975). Nocardiosis. A review of twenty-five cases occurring during twenty-four months. Mayo Clinic Proceedings, 50, 657-663.

Goodman, J. S., and Koenig, M. G. (1970). Nocardia infections in a general hospital. Annals of the New York Academy of Sciences, 174, 552-567.

Hoeprich, P. D., Brandt, D., and Parker, R. H. (1968). Nocardial brain abscess cured with cycloserine and 
sulfonamides. American Journal of Medical Science, 255, 208-216.

Jacobson, J. R., and Cloward, R. B. (1948). Actinomycosis of the central nervous system: a case of meningitis with recovery. Journal of the American Medical Association, 137, 769-771.

King, R. B., Stoops, W. L., Fitzgibbons, J., and Bunn, P. (1966). Nocardia asteroides meningitis: a case successfully treated with large doses of sulfadiazine and urea. Journal of Neurosurgery, 24, 749751.

Kremer, E. P. (1972). Pulmonary and cerebral nocardial abscess. Medical Journal of Australia, 2, 538-540.

Krick, J. A., Stinson, E. B., and Remington, J. S. (1975). Nocardia infection in heart transplant patients. Annals of Internal Medicine, 82, 18-26.

Krueger, E. G., Norsa, L., Kenney, M., and Price, P. A. (1954). Nocardiosis of the central nervous system. Journal of Neurosurgery, 11, 226-233.

Larsen, M. C., Diamond, H. D., and Collins, H. S. (1959). Nocardia asteroides infection: a report of seven cases. Archives of Internal Medicine, 103, $712-725$

Lee, C., Dorman, D. C., and Bradhurst, P. G. (1963). Disseminated nocardiosis: an acute fatal episode in a young girl. A ustralian Medical Journal, 2, 536-541.

List, C. F., Williams, J. R., Beeman, C. B., and Payne, C. A. (1954). Nocardiosis with multilocular cerebellar abscess. Journal of Neurosurgery, 11, 394-402.

Lott, T., El Gammal, T., Dasilva, R., Hanks, D., and Reynolds, J. (1977). Evaluation of brain and epidural abscesses by computerised tomography. Radiology, 122, 371-376.

Maderazo, E. G., and Quintiliani, R. (1974). Treatment of nocardial infection with trimethaprim and sulfamethoxazole. American Journal of Medicine, 57, 671-675.

Munslow, R. A. (1954). Actinomycotic (Nocardia asteroides) brain abscess with recovery: case report. Journal of Neurosurgery, 11, 399-402.

Murray, J. F., Finegold, S. M., Froman, S., and Will, D. (1961). The changing spectrum of no- cardiosis: a review and presentation of nine cases. American Review of Respiratory Diseases, 83, 315 330.

Neu, H. C., Silva, M., and Haxen, E. (1957). Necrotizing nocardial pneumonitis. Annals of Internal Medicine, 66, 274-284.

Pizzolato, P., Ziskind, J., Derman, H., and Buff, E. E. (1961). Nocardiosis of the brain: report of three cases. American Journal of Clinical Pathology, 36, 151-156.

Poretz, D. M., Smith, M. N., and Park, C. H. (1975). Intracranial suppuration secondary to trauma infection with nocardia asteroides. Journal of the American Medical Association, 232, 730-731.

Presant, C. A., Wiernik, P. H., and Serpick, A. A. (1973). Factors affecting survival in nocardiosis. American Review of Respiratory Diseases, 108, 1444-1448.

Schiefer, W., and Huk, W. (1976). Computerised tomographic findings with brain abscess. (1976). In Cranial Computerised Axial Tomography, pp. 360-363. Edited by W. Lanksch, and Kazner, E. Springer-Verlag: Berlin.

Shuster, M., Klein, M. M., Pribor, H. C., and Kozub, W. (1967). Brain abscess due to nocardia: report of a case. Archives of Internal Medicine, 120, 610-614.

Stevens, H. (1953). Actinomycosis of the nervous system. Neurology (Minneapolis), 3, 761-772.

Teleghani-Far, M., Barber, J. B., Sampson, C., and Harden, K. A. (1964). Cerebral nocardiosis and alveolar proteinosis. American Review of Respiratory Diseases, 89, 561-565.

Turner, E., and Whitby, J. S. (1969). Nocardial cerebral abscess with systemic involvement successfully treated by aspiration and sulfonamides: case report Journal of Neurosurgery, 31, 227-229.

Turner, O. A. (1954). Brain abscess caused by nocardia asteroides. Journal of Neurosurgery, 11, 312-318.

Young, L. S., Armstrong, D., and Bleuins, A. (1971). Nocardia asteroides complicating malignant disease. American Journal of Medicine, 50, 356-367. 